\title{
Evaluating the impact of sampling strategies and bioinformatics on ethanol-based DNA metabarcoding
}

\author{
Filipa MS Martins屯,§, Nuno A Fonseca§, Bastian Egeterl, Joana Pinto§, Tiago Assunçãođ, Cátia Chaves \\ $\S$, Pedro Sousa ${ }^{\S}$, Joaquim Jesus ${ }^{\mathbb{T}, \#}$, Pedro Beja ${ }^{\S, n}$ \\ ‡ Departamento de Biologia, Faculdade de Ciências da Universidade do Porto, Porto, Portugal \\ $\S \mathrm{CIBIO/InBio,} \mathrm{Centro} \mathrm{de} \mathrm{Investigação} \mathrm{em} \mathrm{Biodiversidade} \mathrm{e} \mathrm{Recursos} \mathrm{Genéticos,} \mathrm{Universidade} \mathrm{do} \mathrm{Porto,} \mathrm{Vairão,} \mathrm{Portugal}$ \\ | Nature Metrics Ltd, Egham, United Kingdom \\ I LEF, Laboratório de Ecologia Fluvial, Universidade de Trás-os-Montes e Alto Douro, Vila Real, Portugal \\ \# CITAB, Centro de Investigação e Tecnologias Agroambientais e Biológicas, Universidade de Trás-os-Montes e Alto Douro, \\ Vila Real, Portugal \\ - ClBIO/InBio, Centro de Investigação em Biodiversidade e Recursos Genéticos, Instituto Superior de Agronomia, \\ Universidade de Lisboa, Lisboa, Portugal
}

Corresponding author: Filipa MS Martins (filipamsmartins@cibio.up.pt)

Received: 20 Feb 2021 | Published: 04 Mar 2021

Citation: Martins FM, Fonseca NA, Egeter B, Pinto J, Assunção T, Chaves C, Sousa P, Jesus J, Beja P (2021)

Evaluating the impact of sampling strategies and bioinformatics on ethanol-based DNA metabarcoding. ARPHA Conference Abstracts 4: e64708. https://doi.org/10.3897/aca.4.e64708

\begin{abstract}
Recent developments on ethanol-based DNA (etDNA) metabarcoding have shown that it is possible to extract meaningful information about macroinvertebrate community diversity and composition from the ethanol used to preserve bulk samples. The major advantages of this molecular approach are the reduced processing time and costs, and the possibility to keep specimens intact for other experiments. Yet, organisms with highly sclerotised exoskeleton or that are rare in the sample have been found to release a lower amount of DNA into solution and tend to be consistently missed by etDNA metabarcoding, thereby compromising the viability of the method. Few studies have shown that the first steps of the metabarcoding workflow are crucial for the good performance of etDNA-based assays, such as the decision on storage time before sampling and the ethanol phase to be analysed, the inclusion of pre-treatment strategies (i.e., freezing), and the choice of the DNA extraction protocol. In this study, we aimed to evaluate the combined effect of various
\end{abstract}


technical choices on the performance of etDNA metabarcoding, considering factors such as sample volume, ethanol phase of sorted and unsorted samples, pre-capture treatments (evaporation vs filtration) and bioinformatic pipelines. Through the application of decisiontree models, our preliminary data revealed that the increase of volume (by itself) is enough to improve PCR amplification yields and proportion of families matching the morphological identifications, with great impact on the detection of hard-bodied and cased taxa. Also, no major differences among phases with or without a sorting step nor among bioinformatic pipelines were detected, particularly at higher volumes. Our results suggest that the higher performance (with lower observed variation) in taxonomic detection at higher volumes is likely a consequence of a higher availability of longer fragments of DNA in solution. This study highlights the importance of understanding the impact of technical choices to improve the efficiency of a DNA-based method, and reinstates etDNA metabarcoding as a potential method in the context of biomonitoring.

\section{Keywords}

freshwater macroinvertebrates, molecular biomonitoring, morphological identification, storage ethanol, Water Framework Directive

\section{Presenting author}

Filipa MS Martins

\section{Presented at}

1st DNAQUA International Conference (March 9-11, 2021) 